\title{
Comparative characteristics of barley hybrids by the anthocyanins content in grain
}

\author{
Kukoeva T.V. ${ }^{1}$, Glagoleva A.Y. ${ }^{1}$, Shoeva O.Y. ${ }^{1}$, Grigoriev Y.N. ${ }^{1}$, Strygina K.V. ${ }^{2}$, \\ Khlestkina E.K. ${ }^{1,2}$ \\ ${ }^{1}$ Institute of Cytology and Genetics, SB RAS, Novosibirsk, Russia \\ ${ }^{2}$ N.I. Vavilov All-Russian Research Institute of Plant Genetic Resources (VIR), Saint-Petersburg, Russia \\ * email: kukoeva@bionet.ru
}

Barley (Hordeum vulgare L.) is one of the most important crop for food industry. To date, the improvement of grain crops nutritional value by the biologically active compounds - anthocyanins, is a worldwide tendency. Therefore, the need to develop an initial selection material for subsequent obtaining barley varieties with an increased anthocyanins content is becoming more tangible. In barley, these compounds can accumulate in the pericarp, where their biosynthesis is controlled by complementary genes $H v A n t 1$ and $H v A n t 2$ mapped to the chromosomes $7 \mathrm{H}$ and $2 \mathrm{H}$, respectively, and/or in the aleurone layer, controlling by the $H v M y c 2$ gene located on the chromosome $4 \mathrm{H}$. In this work, using the marker-assisted selection, the barley hybrid lines carrying $H v A n t 1, H v A n t 2$ and $H v M y c 2$ genes, based on Siberian barley varieties Aley, Tanay and Vorsinsky 2 were obtained. The near-isogenic barley lines (NILs): PLP (purple lemma and pericarp, carrying Antl and Ant 2 genes) and BA (blue aleurone, carrying $H v M y c 2$ gene) from NordGen (www.nordgen.org) were used as donors. The selection of homozygous plants from the $\mathrm{F}_{2}$ populations was carried out with the intragenic PCRand CAPS-markers. From the selected plants nine hybrids of $F_{2}$ generation with the following phenotypes were taken for lines development: three hybrids with purple pericarp and hulled grain, three hulled and three naked hybrids with blue aleurone. To date, the $\mathrm{F}_{8}$ generation of selected plants was obtained. The quantitative spectrometric analysis of anthocyanins content in the whole grain showed that the most obtained hybrids have a significantly increased anthocyanin content in grain in compare with parental NILs. In hybrids obtained by the BA x Tanay crossing the anthocyanin content was in three times higher than in BA line. Thus, the developed lines are an appropriate material for further selection of barley varieties enriched with anthocyanins.

Acknowledgments: The study was supported by the RFBR grant No. 19-016-00140. 